\title{
Oblique split technique: a game changer in costal cartilage sculpting
}

\author{
Fazil Apaydin \\ Department of Otorhinolaryngology, Bornova, Izmir 35100, Turkey. \\ Correspondence to: Prof. Dr. Fazil Apaydin, Department of Otorhinolaryngology, Bornova, Izmir 35100, Turkey. \\ E-mail: fazil.apaydin@ege.edu.tr
}

How to cite this article: Apaydin F. Oblique split technique: a game changer in costal cartilage sculpting. Plast Aesthet Res 2019;6:9. http:// dx.doi.org/10.20517/2347-9264.2018.73

Received: 10 Oct 2018 First Decision: 18 Mar 2019 Revised: 27 Mar 2019 Accepted: 29 Mar 2019 Published: 26 Apr 2019

Science Editor: Hong Ryul Jin Copy Editor: Cai-Hong Wang Production Editor: Huan-Liang Wu

\begin{abstract}
Oblique split method is a technique used to carve the costal cartilage. Its main advantages are the high number of grafts that can be obtained, the ability to obtain grafts of various thicknesses and lengths, no risk of warping, less chance of desorption, preservation of the straight forms of grafts although they can be carved in different shapes.
\end{abstract}

Keywords: Oblique split method, saddle nose, structural rhinoplasty, revision rhinoplasty, septal reconstruction

\section{INTRODUCTION}

Costal cartilage is one of the main sources for grafts in the reconstruction of saddle nose deformities and revision rhinoplasty cases. However, its handling and carving methods have always created a challenge for the surgeon. In 1958, Gibson and Davis published a technique called as "principle of the balanced crosssection" which could be used to overcome warping, the major disadvantage of the use of carved/sculpted costal cartilage ${ }^{[1]}$. They stated that if the distorting forces were balanced along a cartilage graft, the grafts would not be distorted (warp). In total 46 balanced cross-section grafts were followed for over a three-year period. The oblique split method after Taştan et al. ${ }^{[2]}$ describes the angle to the long axis of the rib cartilage upon which the costal cartilage is cut/sectioned. In this original description was there was no clinical observation of graft warping in the follow-up period. It was also stated that although the grafts obtained could be modified into different shapes, they preserved their straight shape.

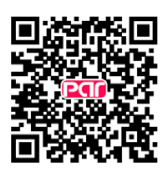



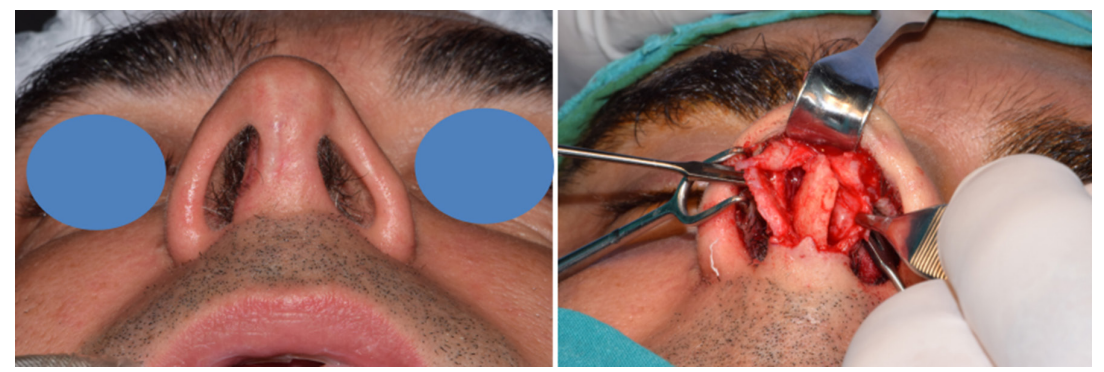

Figure 1. In the reconstruction of a crooked nose, a subtotal reconstruction was done by L-strut obtained from the 6th rib. It had warped and a revision was needed

\section{METHODS}

\section{Costal cartilage sculpting methods}

The principle of the balanced cross-section

This principle is still the fundamental technique when costal cartilage use comes into consideration in saddle noses corrections or revision rhinoplasties ${ }^{[1]}$. The sculpted cartilage grafts are used as structural grafts and augmentation grafts. Besides, the integrated dorsal graft/columellar strut has been used in many cases where the caudal septal support was poor ${ }^{[3]}$. It has been reported that careful symmetric carving of costal cartilage could minimize the chance of cartilage warping over time ${ }^{[3]}$. Our experience has shown us that costal cartilage warping can still occur despite careful symmetric carving, especially while using structural grafts [Figure 1]. The one-piece L-strut graft obtained from the costal cartilage was described by Rettinger and its use has been effective for decades ${ }^{[4]}$. Our experience of dorsal onlay grafts is that they rarely have clinically apparent warping. However, warping has remained the primary concern with the use of costal cartilage grafts. Many cutting and additional techniques have been developed to overcome this issue. For the last 19 years we have been utilizing balanced cross-sectional carving and although warping was rarely seen, concerns always remained. The use of thicker grafts employed by us and other experienced rhinoplasty surgeons to minimize warping resulted in stiffer noses, which was undesirable to some patients.

Freehand carving/sculpting of costal cartilage with a scalpel has historically been the technique of choice for fashioning grafts. The creation of thin grafts using the technique is however technically challenging and carries a higher risk of warping. Dermatome blades overcome the technical challenges of producing thin grafts, the unfavorable warping characteristics however remains ${ }^{[5]}$.

An observed major limitation was the paucity of grafts that can be obtained by this method.

The central portion of the rib is utilized as a graft, with the remaining peripheral cartilages portions/ shavings often unusable due to the unfavorable warping characteristics. Typically in the correction of saddle nose deformity, we use the central rib portion for caudal septal graft and the one outer layer/shaving for dorsal onlay graft. However, in revision rhinoplasties variation in graft type, size and amount graft material required limit the use of balanced cross-section carving rib carving.

\section{Oblique split method}

I have learned this technique during a meeting from Dr. Taştan in 2008, several years prior to its publication ${ }^{[2]}$. Impressed with the simple logical solution to this common and difficult problem I adopted his technique. My preferred ribs have been the 5th and the 6th due to the ease of access through the inframammary incision in females. After harvesting the 4 to $6 \mathrm{~cm}$ long rib, the cutting angles, direction and the length of the implants are calculated dependent on the requirements. The idea is to obtain the longest possible implants depending on the shape of the harvested cartilage [Figure 2]. 

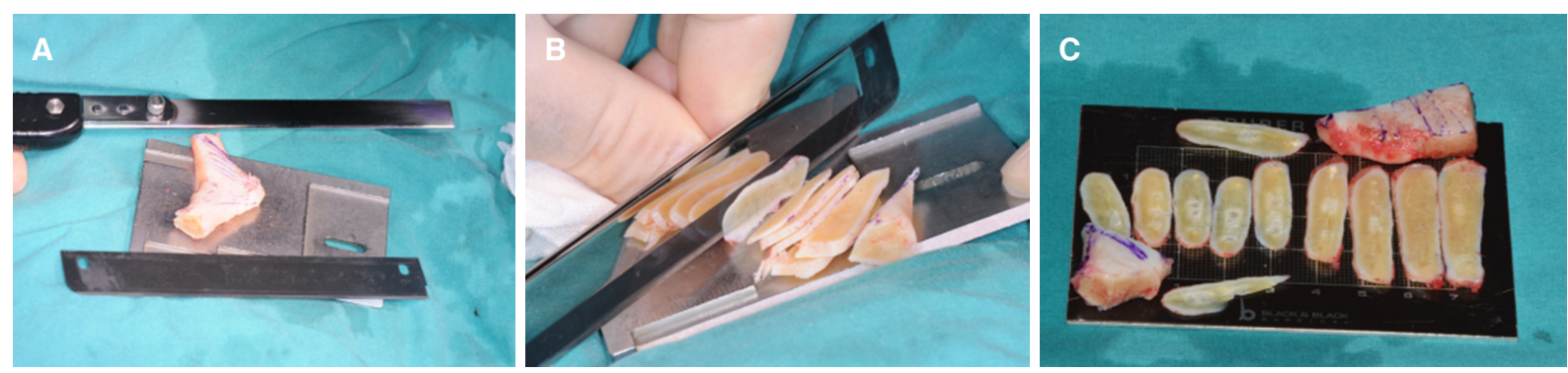

Figure 2. The sixth costal cartilage is obtained and markings performed to decide on the best way for oblique cuts ( $A$ ); The Chef's knife is used for precise cuts (B); As seen in the picture, about 12 implants of various thicknesses with the preserved outer cortex are obtained and more implants can be sculpted from the remaining cartilage (C)
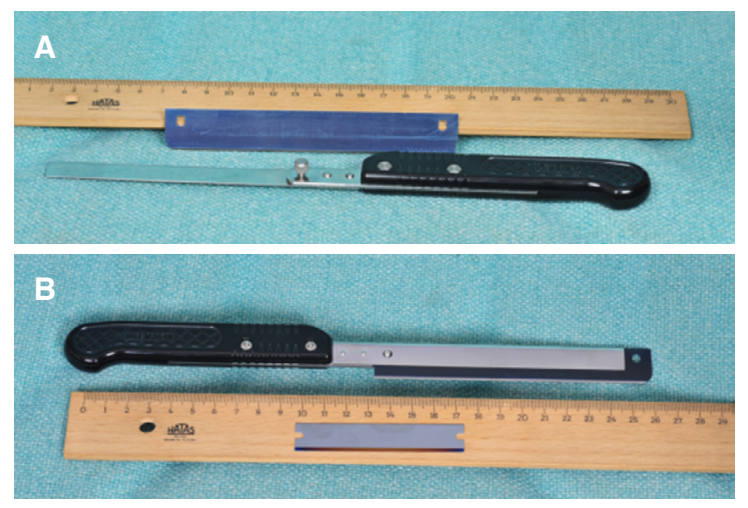

Figure 3. The Chef's knife is composed of two parts: a $26 \mathrm{~cm}$ blade holder and a $13 \mathrm{~cm}$ blade (A). The blade is inserted in the housing of the holder and a bolt is used to fix the blade in place. The microtome blade is put on the ruler for comparing the dimensions of both blades (B)

What kind of instruments have I used throughout the past 10 years? I started using the microtomes of the pathologists. They were longer than a scalpel and very helpful to make clean long cuts and obtain smooth grafts. After cutting my fingers a few times, I found a microtome handle which kept my fingers on the safe side. Then I used dermatome blades which further improved sectioning the rib cartilage. Almost four years ago, I found the ideal blade which I call as Chef's knife ${ }^{[6]}$. While working with the costal cartilage, I always looked for a knife with the cutting height of at least 7-8 $\mathrm{mm}$ which is usually the height of the rib. The blades are $13 \mathrm{~cm}$ in length and $14 \mathrm{~mm}$ in height. When placed in the blade holder, it's cutting height becomes $8 \mathrm{~mm}$ [Figure 3]. With the help of this knife, the cuts can be done more precisely with improved control, and 0.5, 1,2 and $3 \mathrm{~mm}$ thick grafts can be obtained. In the majority of the cases, the outer cortex is left intact which helps stability and prevents warping and absorption.

\section{Clinical situations to use costal cartilage}

Nasal septum

Nasal septum can be severely deviated, or partially absent especially in saddle nose, congenital disorders such as Binder's syndrome or cleft lip nose. The severe deviation can be traumatic or iatrogenic due to previous surgery. In these situations, there is a need for straight implants to reconstruct the nasal septum. The nasal septum can be divided into imaginary sections, and these grafts can be used to reinforce or replace each of the segments (dorsal and or caudal) to obtain a straight L-strut. In cases of previous septal abscess where all the septal cartilage is deficient, it is possible to reconstruct the entire septal cartilage by using the grafts obtained with an oblique cutting technique. Instead of using a template such as PDS foil, these implants of 1-2 $\mathrm{mm}$ in thickness can be sutured on $0.5 \mathrm{~mm}$ thick implants. The dorsal segment can be supported from both sides by splinting spreader grafts, then they can be coupled with a caudal septal extension or replacement grafts [Figure 4]. 

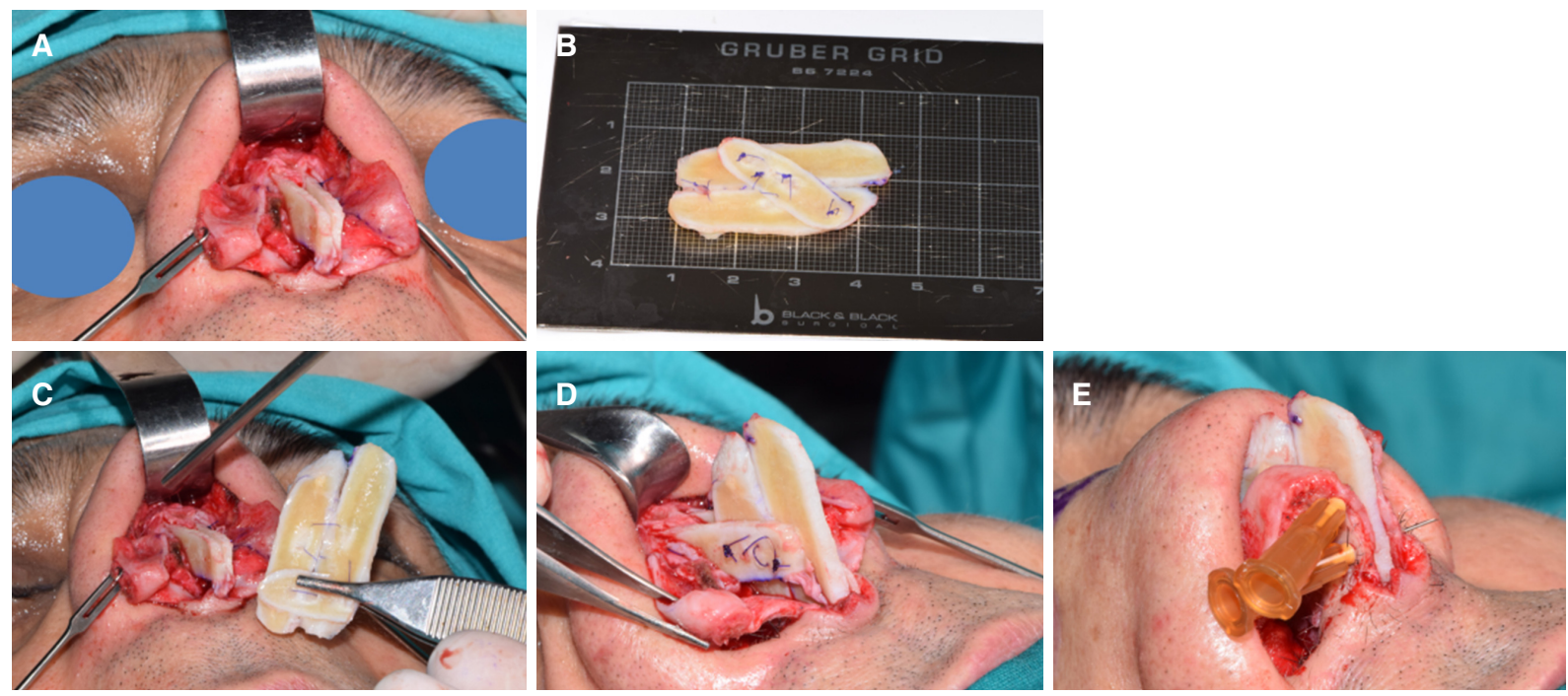

Figure 4. Two splinting spreader grafts are sutured to the small piece of septal cartilage at the key area (A); a new septum is constructed by bringing two $2 \mathrm{~mm}$ thick implants sutured to each other by $0.5 \mathrm{~mm}$ thick graft (B, C); the new septum is sutured to the spreader grafts to rebuild the L-strut (D); the medial crura are sutured to the new septum as a tongue-in-groove (E)
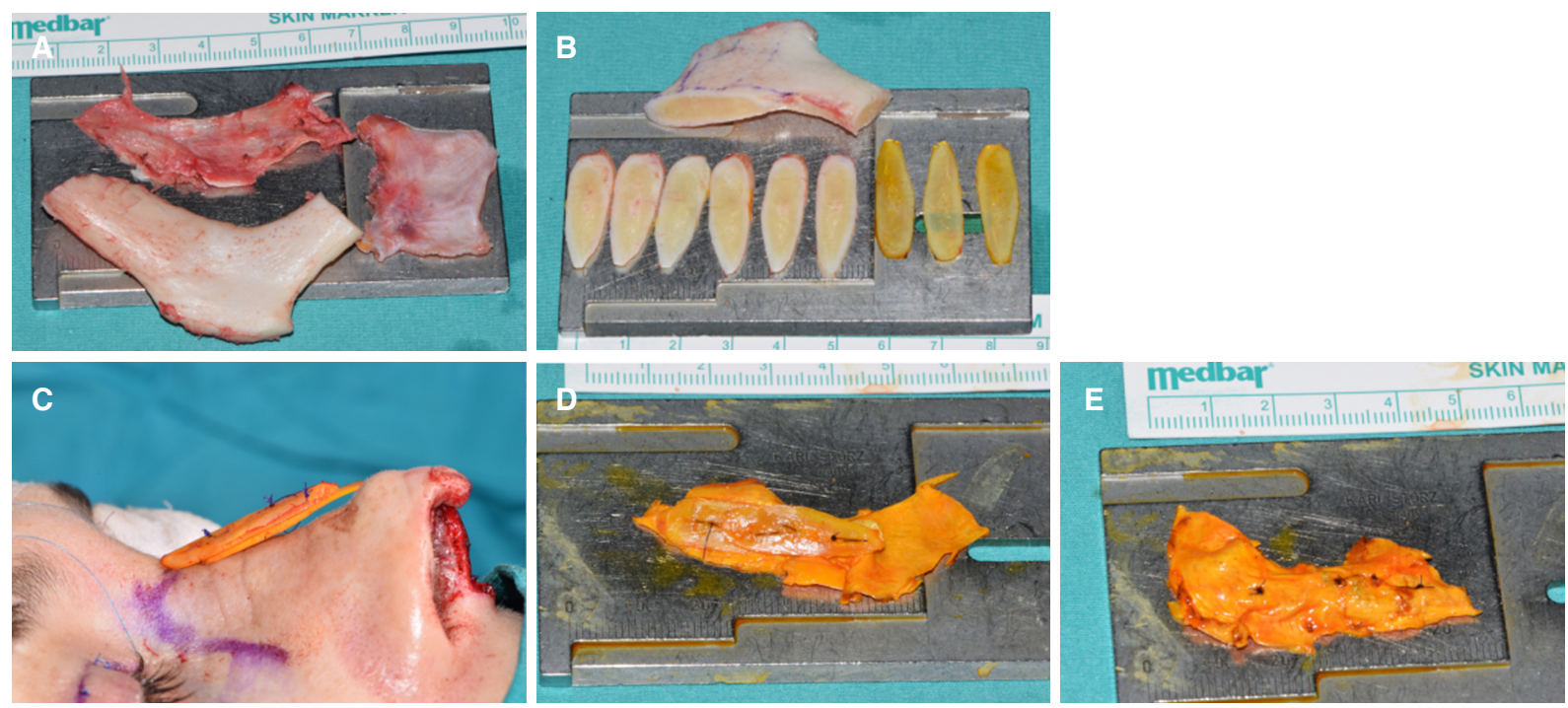

Figure 5. The sixth rib harvested with rectus abdominis fascia and the outer perichondrium (A); multiple implants of various thicknesses are cut by oblique split (B); two laminas are sutured together for augmentation (C); the dorsal surface and the lateral parts of the implants are covered by perichondrium for camouflage $(D, E)$

\section{Saddle nose}

In saddle noses, there are two main issues to address: the nasal septum and the need for augmentation. Regarding the septum, the technics are described above. For dorsal augmentation, I have been using the following techniques: (1) Solid onlay grafting; (2) Laminated grafts; (3) Diced cartilage in fascia ${ }^{[7,8]}$; (4) Cartilage chips in fascia.

The Laminated graft technique involves the combining of two or more obliquely cut grafts by suturing. They are tailored to the needs of the augmentation by partial shaving. I like covering the upper part by means of perichondrium obtained from the outer surface of the rib [Figure 5]. The advantage of this technique is that many oblique cut grafts can be brought together to get the desired dimensions with no risk of warping and resorption. 

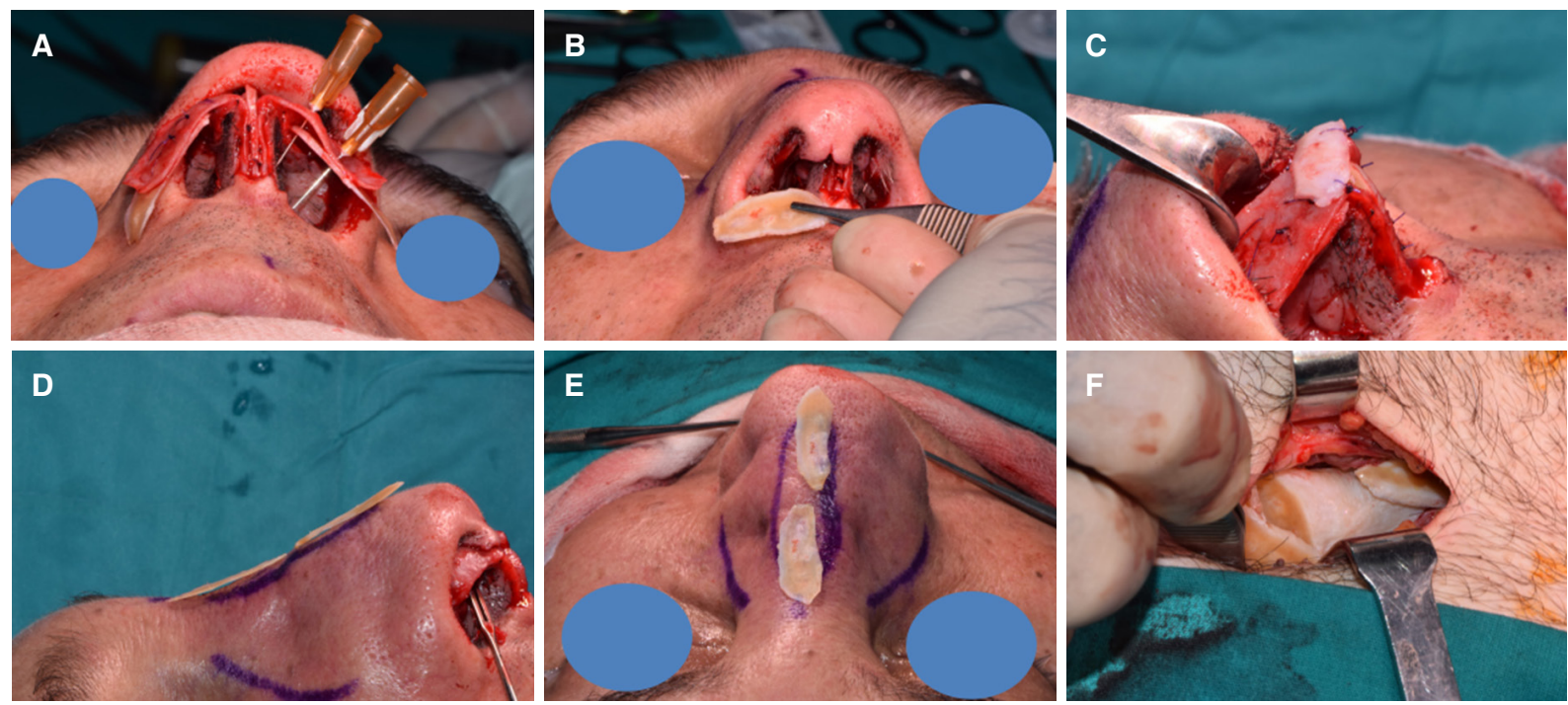

Figure 6. When thinner implants are used as lateral crural strut grafts, the patients do not palpate them from the inside (A); a similar situation is valid for alar batten grafts as well (B); tip grafts can easily be carved from rib cartilage (C); paper thin implants can be used for camouflage purposes $(D, E)$; the remaining cartilages can be inserted into the recipient bed for support $(F)$
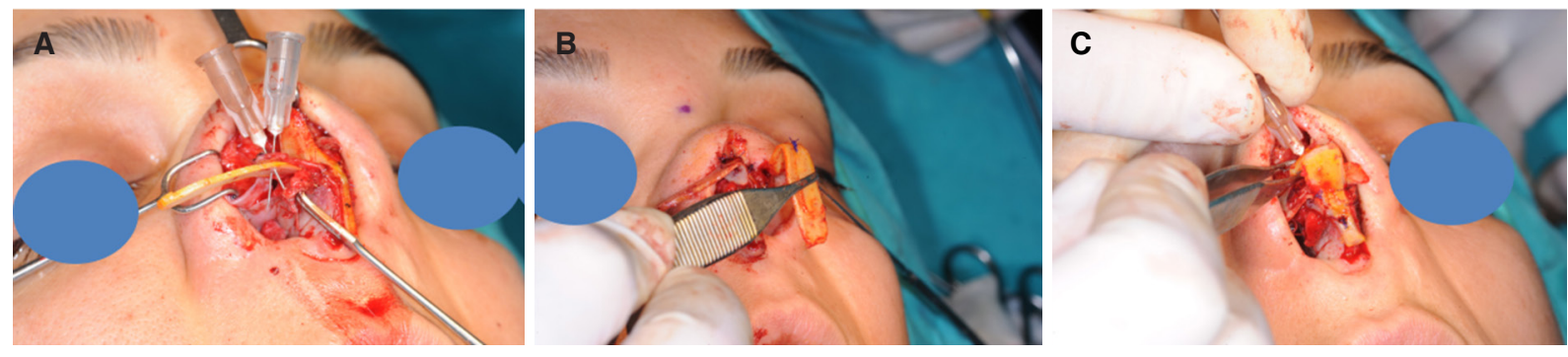

Figure 7. In younger patients, the thin implants are flexible enough to reconstruct the missing lower lateral cartilages partially or totally. In this case, the lateral crura and the domes are reconstructed by thin rib implants (A, B); then a shield graft is sculpted for a better tip definition and projection (C)

\section{Revision rhinoplasty}

The patients usually seek revision surgery due to over resection, under resection or persistent nasal deviation. In cases of over resection, graft requirements can often exceed the amounts of cartilage that can be harvested from conchal cartilages. In these situations, the oblique split technique has provided large amounts of straight grafts of various thicknesses and lengths from a single rib, while avoiding technically complicated and time consuming carving techniques. The volume and the variability grafts that can be produced combined with the speed of the oblique split method affords the surgeon greater flexibility than other methods. I have had a chance to cut paper thin implants to use as lateral crural strut grafts and camouflage grafts [Figure 6]. In younger patients, the thinner implants can easily be bent to reconstruct the lower lateral cartilages [Figure 7]. These thinner grafts avoid rigidity and stiffness associated with the use of the thicker grafts.

In revision rhinoplasties and saddle noses, a very useful technique that I have used over the last 5 years is to put paper-thin slices of cartilage, called as cartilage chips, within rectus abdominis fascia or temporalis fascia. In my experience, this is a superior technique than diced cartilage in fascia (a technique which I have used for 8 years), because although very similar in purpose, it stays much firmer on the dorsum than diced cartilage, so the patient is not able to make any changes with the shape of the dorsum [Figure 8]. 

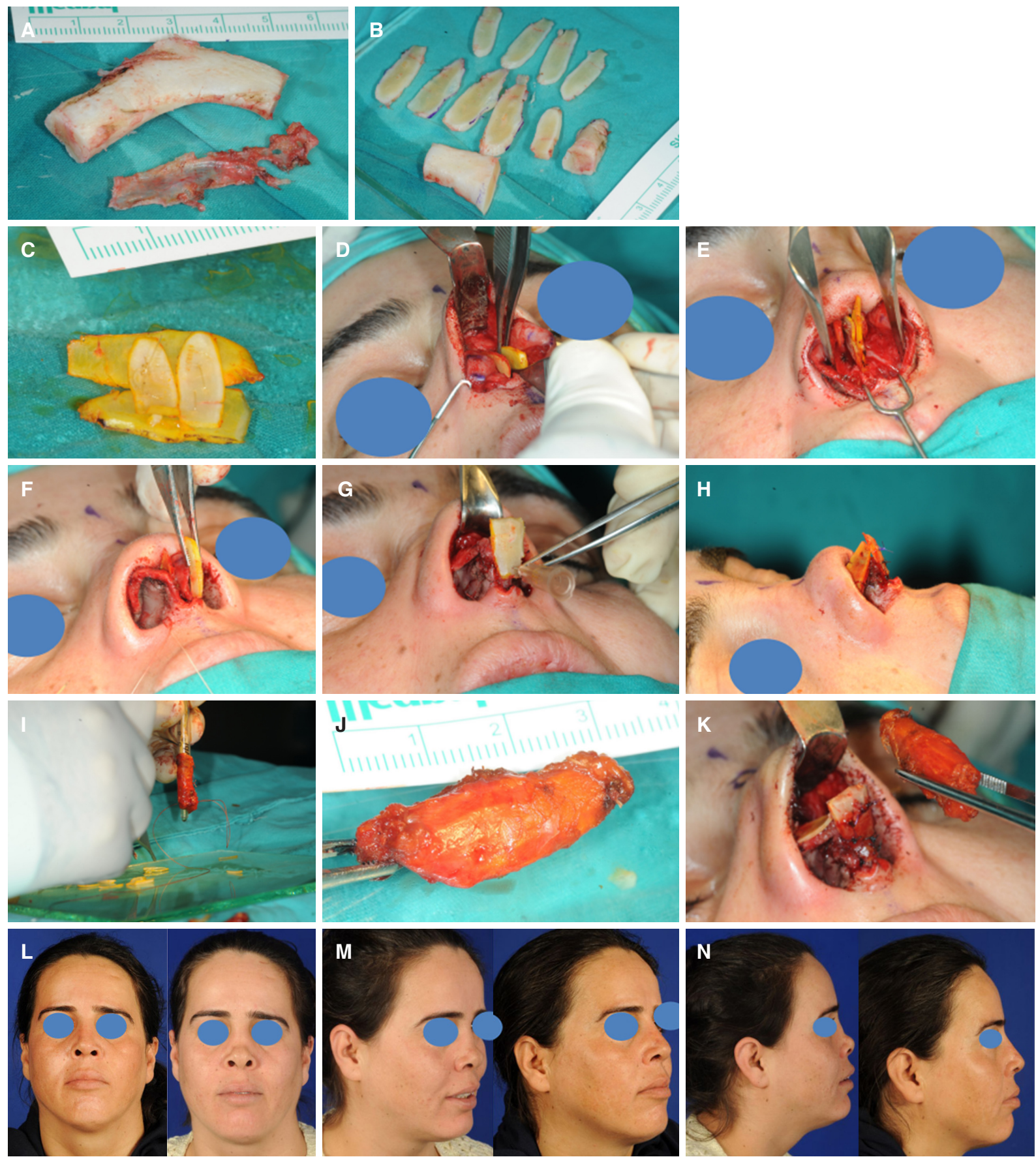

Figure 8. A 28-old female patient had nasal trauma followed by a septal abscess four years ago. She has ended with a short saddle nose. During examination, the septal cartilage was missing. The sixth rib was harvested and obliquely cut (A, B); a new septum was formed (C) and sutured to the splinting spreader grafts $(D, E)$; a columellar strut, shield graft and lateral crural onlay grafts were used $(F, G, H)$; cartilage chips were inserted in rectus abdominis fascia $(I, J, K)$; after two years, the patient was happy with the result $(L, M, N)$

\section{CONCLUSION}

There are two main types of costal cartilage sculpting: symmetrically balanced cross-section and oblique split method. The author's clinical experience over the past decade has demonstrated the superiority of the oblique split method in terms of graft material and reduction in clinical significant warping. 


\section{DECLARATIONS}

\section{Authors' contributions}

The author contributed solely to the article.

\section{Availability of data and materials}

Not applicable.

\section{Financial support and sponsorship}

None.

\section{Conflicts of interest}

The author declared that there are no conflicts of interest.

\section{Ethical approval and consent to participate}

An informed consent from the patients to participate in the study has been taken.

\section{Consent for publication}

An informed consent for publication has been taken from the patients.

\section{Copyright}

(c) The Author(s) 2019.

\section{REFERENCES}

1. Gibson T, Davis WB. The distortion of autogenous cartilage grafts: Its cause and prevention. Br J Plast Surg 1958;10:257-74.

2. Taştan E, Yücel ÖT, Aydin E, Aydoğan F, Beriat K, et al. The oblique split method: a novel technique for carving costal cartilage grafts. JAMA Facial Plast Surg 2013;15:198-203.

3. Kim DW, Toriumi DM. Management of posttraumatic nasal deformities: the crooked nose and the saddle nose. Facial Plast Surg Clin North Am 2004;12:111-32.

4. Rettinger G. Reconstruction of the pronounced saddle nose. Laryngorhinootologie 1997;76:672-5. (in German)

5. Foulad A, Hamamoto A, Manuel C, Wong BJ. Precise and rapid costal cartilage graft sectioning using a novel device: clinical application. JAMA Facial Plast Surg 2014;16:107-12.

6. Apaydin F. The "Chef's Knife" in oblique split technique for rhinoplasty. JAMA Facial Plast Surg 2015;17:382-3.

7. Erol OO. The Turkish delight: a pliable graft for rhinoplasty. Plast Reconstr Surg 2000;105:2229-41; discussion 2242-3.

8. Daniel RK, Calvert JW. Diced cartilage grafts in rhinoplasty surgery. Plast Reconstr Surg 2004;113:2156-71. 